\title{
Nonlinear mechanics of flexible cables in space robotic arms subject to complex physical environment
}

\author{
Yuwang Liu(D) - Jibiao Chen · Jinguo Liu • \\ Xingjian Jing
}

Received: 11 February 2018 / Accepted: 23 May 2018 / Published online: 8 June 2018

(C) The Author(s) 2018

\begin{abstract}
A nonlinear model of a special cable in space robotic arms is developed in space environment. The mechanic effects of control cables in powerful robots can often be neglected. However, in complex space multi-physics environments, involving ultra-low temperature, radiation, and other extreme conditions of outer space, the externally mounted cables (protected by shielding layers) can induce strong nonlinear interference to robot arms; and this can induce further small-range slow rotations or oscillations of the flexible joint of robots at a specific posture, which consequently affect the precision and operation performance of end effectors. Effective mathematical models on nonlinear mechanics of strong cables under multi-physics environments and their effects on weak robots have not been well developed yet. Complex key factors, such as low gravity, nonlinear friction, and unexpected curved surface constraints, have not been extensively investigated either. In this study, considering all these key factors, a Kirchhoff nonlinear mechanical model of cables in

Y. Liu $(\varangle) \cdot$ J. Chen $\cdot$ J. Liu

State Key Laboratory of Robotics, Shenyang Institute of Automation, Chinese Academy of Sciences, Shenyang, People's Republic of China

e-mail: liuyuwang@sia.cn

J. Chen

Department of Mechanical Engineering and Automation, Northeastern University, Shenyang,

People's Republic of China

X. Jing

Department of Mechanical Engineering, The Hong Kong Polytechnic University, Hong Kong, People's Republic of China
\end{abstract}

complex space environments is developed, and a relatively improved algorithm based on a trust-region strategy is proposed for solving this nonlinear model, based on which the geometry and terminal force of the modeled robot cable can be obtained. The validity and accuracy of the proposed algorithm and theoretical calculation results are verified via experiments. The theoretical findings revealed in this study are significant to future research on the slow rotations and oscillations of weak robot joints in space exploration with robotic arms.

Keywords Strong nonlinear interference - Slow rotation of joints - Kirchhoff nonlinear mechanics . Curved surface contact

\section{Introduction}

The complex nonlinear mechanical characteristics of control cables could be critical to the stability and accuracy of the joint operation of robot arms. Flexible cables, which are employed for various functions including power supply and information transmission, are indispensable parts of robotic systems. In general, the internal and external flexible cables employed in robot arms are relatively very weak compared to expected robotic payload and output torques. Their nonlinear mechanical properties can usually be negligible consequently. However, considering space environments, including ultra-low temperature, radiation, and other extreme environment of outer space, coin- 
cided with resource constraints, the precision manipulator joint is often relatively weak with limited output torque. Moreover, in order to preserve communication security and provide a reliable supply of power, flexible cables are often wrapped with thick nonmetallic shielding layers which can become exceptionally rigid under the conditions of long-term radiation or a cold environment. Consequently, externally mounted cables protected by shielding layers can induce strong nonlinear interference effects on robot arms. Owing to this interference, the flexible joints of robot arms could slowly rotate or oscillate within a small range when the robot arm is in a certain posture; this significantly affects the precision and operation performance of the end effectors of robots. It is thus very relevant to study the nonlinear mechanics of flexible cables in space robots under the conditions of multi-physics coupling dynamics. Robots employed for planetary explorations are often exposed to special gravity fields and placed in extremely limited space. Gravity, friction, and surface geometric constraints are often the key factors that need to be studied to determine the mechanical properties of cables.

In recent years, flexible bodies including cables have been extensively investigated in various fields. Payandeh et al. [1] revealed the geometric shape of a surgical suture by using particle-spring model, which accurately represents the tensile deformation of a flexible body that is prone to elastic deformations. Servin et al. [2] proposed a connecting rod chain model, i.e., a series of connecting rods, to characterize the cable shape and study the pulling deformation of the cable without considering twisting effects. Lazarus et al. [3] employed quaternions to develop a flexible cable model. In addition, an asymptotic numerical method has been employed to obtain the cable shape; however, this method only considered cable twisting. The above-mentioned methods focused on the shape of the cable. In contrast, a nonlinear mechanical model of an elastic rod can accurately reveal the cable shape and mechanical properties at discrete points along the cable. The Cosserat [4,5] and Kirchhoff [6-14] theories are mainly employed in this type of model. Owing to its simplicity, the Kirchhoff cable mechanics model is currently widely used [15-21]. By implementing the Kirchhoff theory and elastic thin rod static model [22], Liu et al. [23-25] developed a cable mechanical model with smooth plane contact to improve the virtual reality assembly technology for cables. Although their model is useful, they ignored the effects of gravity on the cable; moreover, the contact between the cable and curved surface was not considered. Consequently, their model is still insufficient for practical application. A model to describe the contact of DNA molecules with curved surfaces has been proposed in [26]. However, this method does not consider various effects such as gravity, friction, and length, and the flexible body was required to remain in full contact with the curved surface; thus, the method cannot be employed when the flexible body and curved surface are partially restrained. In addition, it can only be employed to solve the initial value problem and cannot be employed to estimate the actual working conditions. Based on previously reported theoretical results [22-26], the effects of gravity and friction on the cable are considered to propose a nonlinear mechanical model of a cable under localized constraints of a curved surface in this paper. When implementing multi-physics coupling methods, the gravity force distribution on the heavy cable, which is determined by the safety shield, is an important factor that should not be neglected. Owing to the limited space resources, it is necessary to consider the friction and geometric constraints between the cable and curved surface. Taking into account the above issues, it is clear that the mechanical constraints on the cable are very complex, the dimensions of the equations increase in size, and the solution of the cable nonlinear mechanics model is even more challenging. In order to ensure global convergence of the iterative calculations, a more reliable solution algorithm is yet to be developed.

Therefore, by considering the gravity, friction, and curved surface constraints, and using a planetary exploration robot as the main object of the model, we employ the Kirchhoff theory to study the joint stability and develop a nonlinear mechanical model of a flexible cable that has one end fixed and other end free. Using the finite difference method, the differential equations are discretized into a set of nonlinear equations, and then transformed into a global optimization problem. In order to ensure global convergence of the iterative calculations, an improved algorithm is proposed based on the trust-region strategy. The large-scale nonlinear optimization problem is solved, and the mechanical parameters of each discrete point of the cable are obtained. For the calculation results, Euler parameters are employed to obtain the coordinates of each discrete point. In addition, a cubic spline curve is used to fit the 
Fig. 1 a Planetary exploration robot. b 2-DOF space observation robotic arm

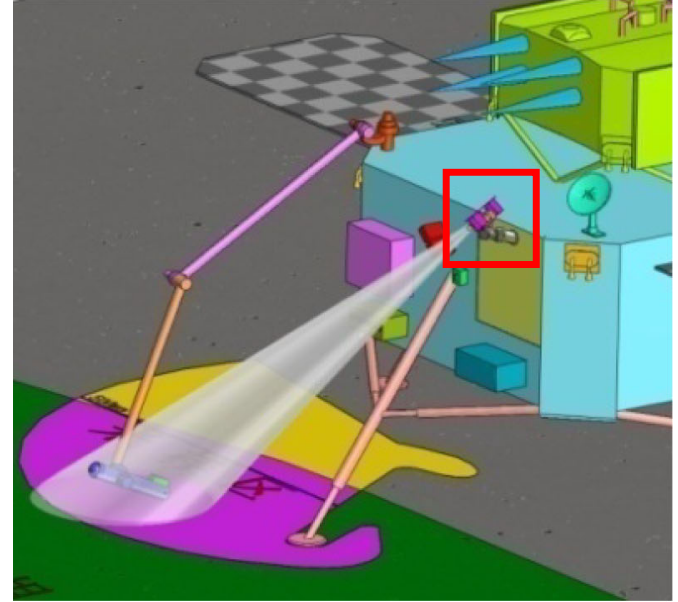

(a)

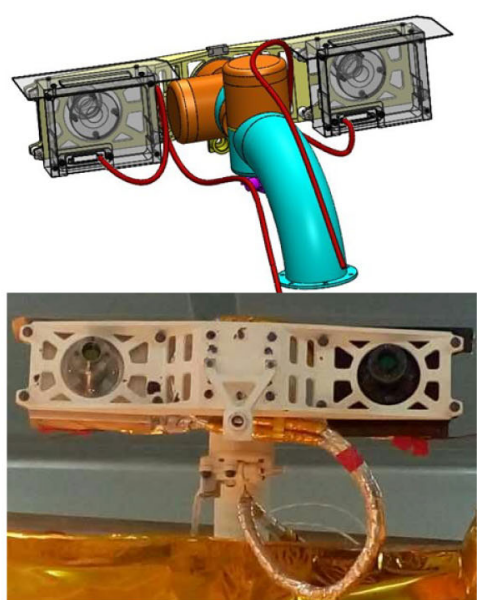

(b) discrete points in order to obtain the geometric shape of the cable in the world coordinate system.

The contributions of this study lie in the following points:

1. First, we introduce and analyze a planetary exploration robot subjected to multi-physics coupling dynamics and consider the space robot joint stability in the presence of strong nonlinear cable interference;

2. Then, we develop a mathematical model of the flexible cable in space robotic arms;

3. Using the developed methods, the mechanical properties and shape of a cable in space robotic arms constrained to a curved surface are revealed.

\section{Analysis of planetary exploration robot designed for multi-physics coupling dynamics}

\subsection{Planetary exploration robot and its cables}

Figure 1a shows an image of the Chinese planetary exploration robot. Once the planetary exploration robot lands on an outer planet, a two-degree-of-freedom (2DOF) robotic arm, as is shown in Fig. 1b, drives two cameras to observe the surface of the planet. In order to ensure normal long-term operation of the cameras at both high and low temperatures in a space radiation environment, the camera cables are wrapped with thick nonmetallic shielding layers. One end of the cables is fixed to the free end of the 2-DOF space observation robotic arm, while the other end is fixed to the base of the 2-DOF observation robotic arm. The camera cables swing according to the movement of the 2-DOF observation robotic arm. At very low temperatures on the surface of the planet under investigation, the nonmetallic shielding layers of the cables become unusually stiff. Furthermore, the nonmetallic shielding layers age and harden with the increase in the space radiation time.

\subsection{2-DOF observation robot joint: slow rotation phenomenon}

As is shown in Fig. 2a, for a single joint, the cable is fixed to the upper and lower links of the joint in the 2-DOF space observation robotic arm, with one end of the joint being installed on the fixed link, whereas the other end is connected to the free link. When the robot moves within a certain range, the cable follows the free end of the joint.

In addition, there are many research results on the error elimination control of flexible structures. A neural network controller is developed to suppress the vibration of a flexible robot system with input deadzone in [34], and the proposed controller has good performance in suppressing vibration. Also, through a boundary controller, a control problem about input backlash in a flexible robotic manipulator is well solved in [35]. A cooperative control with good control effect is proposed for a nonuniform gantry crane (consisting of a flexible cable and other components) with constrained tension in [29]. And an optimal feedback linearization approach is used 


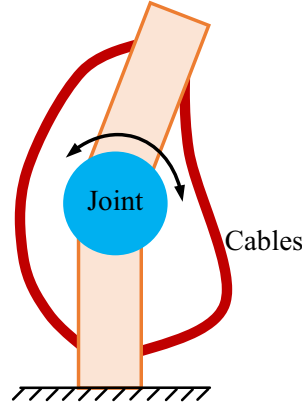

(a)

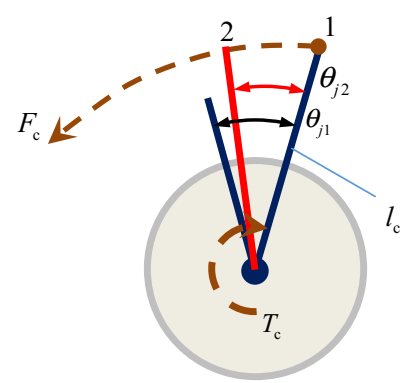

(b)

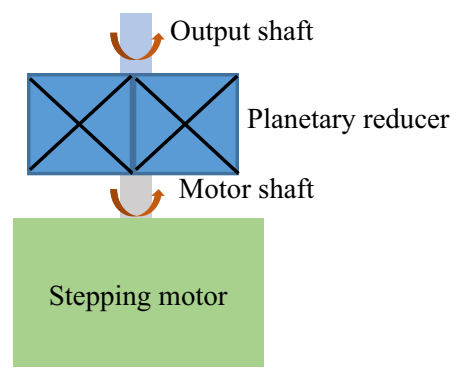

(c)

Fig. 2 Slow rotation of the joint of a 2-DOF space observation robotic arm. a Overall shape of the joint. b Analysis of the force on the joint. c Stepper motor and planetary reducer connection diagram

to find the optimal path between the initial and the target points in the case of a cable robot under the maximum dynamic load. These are very good research results in controlling cable robots in [28,30,31]. Different from the above error elimination algorithms, high-precision position sensors cannot be used at present (due to the strict size constraints and limitations of high and low temperature space environments), the joint rotation can only be open-loop controlled by a stepper motor. Therefore, the problem proposed can only be solved by developing a theoretical cable model instead of using a control algorithm to eliminate the error.

As shown in Figures $2 b$, c, the joint is driven by a single stepper motor through a planetary reducer. The inherent gap $\theta_{j 1}$ (less than 1 degree) of this joint is mainly caused by the planetary reducer. The axis of rotation of the robotic joint is parallel to the direction of the microgravity field.

Defining $T_{\mathrm{m}}$ as the load torque of the joint:

$T_{\mathrm{m}}=F_{\mathrm{c}} l_{\mathrm{c}}-T_{\mathrm{c}}$ where $l_{\mathrm{c}}$ is the arm of $F_{\mathrm{c}}$ with respect to the joint axis. The internal damping torque $T_{\mathrm{c}}$ of the joint includes the bearing friction torque, internal cable resistance torque of the joint, and damping torque induced by the planetary reducer. There is an inherent gap $\theta_{j 1}$ at the joint, and when the joint rotates, the cable force $F_{\mathrm{c}}$ changes nonlinearly. Slow rotation of a robotic joint may occur when the load torque $T_{\mathrm{m}}$ is close to 0 , as is illustrated in Fig. 2b. Because the presence of the gap is evident at a particular position when the load torque $T_{\mathrm{m}}$ is close to 0 . In Fig. 2b, Position 1 is the target stop position of the robotic joint for end-effector operation. Owing to the cable force $F_{\mathrm{c}}$, the joint slowly rotates until it reaches $\theta_{j 2}$, which corresponds to Position 2. Owing to the internal damping of the joint, the joint gap $\theta_{j 2}$ is smaller than the inherent gap $\theta_{j 1}$. Position 2 is the final position after the slow rotation of the joint. Moreover, at Position 2, the torque of the cable force $F_{\mathrm{c}}$, offsets the internal damping torque $T_{\mathrm{c}}$ of the joint with respect to the joint axis; this places the robotic system in equilibrium $\left(T_{\mathrm{m}}=0\right)$. When the load torque $T_{\mathrm{m}}$ is much greater than 0 , under the effect of the load torque $T_{\mathrm{m}}$, the joint will reach Position 2 in a flash, and the slow rotation of a robotic joint will not occur. When $F_{\mathrm{c}}$ tends 0 , the joint will remain motionless under the effect of the internal damping torque $T_{\mathrm{c}}$, the joint will stay at Position 1, and this phenomenon will also not occur.

In [27], although constraints were imposed at both ends, only the influence of the disturbance torque of a single cable on a stabilized platform was studied. However, there are more complex constraints on cables used in the manufacture of robots purposed for outer space missions; furthermore, the robotic joint is influenced by the mechanical characteristics of multiple cables. The strong interference effects induced by the cables are most significant when the joint is in a particular position. The free end of the robot joint is equipped with camera sensors, and the slow rotation of the joint can significantly reduce the quality of the images recorded by the cameras. It is worth noting that there is currently no extensive research being carried out in this area. The mechanical properties of cables implemented in robots are the main reasons for these phenomena. In order to study the above phenomena, we develop and solve a nonlinear mechanical model of a robot-purposed cable under the conditions of a complex outer space environment. 


\section{Mechanical modeling of a flexible cable}

\subsection{Basic assumptions}

We made the following assumptions for the development of a simple cable mechanical model in the 2-DOF space observation robotic arm [22-25]:

1. The cross section of the cable is a rigid circular section, and the cable centerline is always perpendicular to the cable cross section.

2. The centerline of the cable is a smooth curve exceeding 2 nd order; the length of the cable is significantly larger than the diameter of its cross section.

3. The cable is isotropic; there is a linear relationship between stress and strain.

4. The deformations of the cable attributed to stretching and shearing are ignored.

\subsection{Coordinate system transformation}

Three different coordinate systems are employed for the developed cable mechanical model (Fig. 3):

\section{Coordinate system $O-X Y Z$}

The coordinate system $O-X Y Z$ is employed as the world coordinate system. The starting point of the cable centerline corresponds to the origin $O$ of the coordinate system. In this coordinate system, an arc coordinate $s$ is introduced along the cable centerline; any point $P$ at the cable centerline can be determined via the arc coordinate $s$. The vector of the point $P$, which is set with respect to the origin $O$, is denoted as $\boldsymbol{r}$; the vector that corresponds to the point $P_{0}$ is $\boldsymbol{r}_{0}$.

\section{Coordinate system $P-N B T$}

The frame $\{P \mid N, B, T\}$, which can be regarded as a rigid body, follows the movement of the point $P$. The parameters affecting the point $P$ are as follows: tangent vector $\boldsymbol{T}(s)=\mathrm{d} \boldsymbol{r} / \mathrm{d} s$, curvature $\kappa=|\mathrm{d} \boldsymbol{T} / \mathrm{d} s|$, main normal vector $\boldsymbol{N}(s)=\mathrm{d} \boldsymbol{T} /(\kappa \mathrm{d} s)(\kappa \neq 0)$, vice normal vector $\boldsymbol{B}(s)=\boldsymbol{T}(s) \times \boldsymbol{N}(s)$, and torsion $\tau=|\mathrm{d} \boldsymbol{B} / \mathrm{d} s|$. The cable centerline can be obtained via Eq. (2); $\varepsilon$ is the integration variable.

$\boldsymbol{r}(s)=\int_{0}^{s} \boldsymbol{T}(\varepsilon) \mathrm{d} \varepsilon$

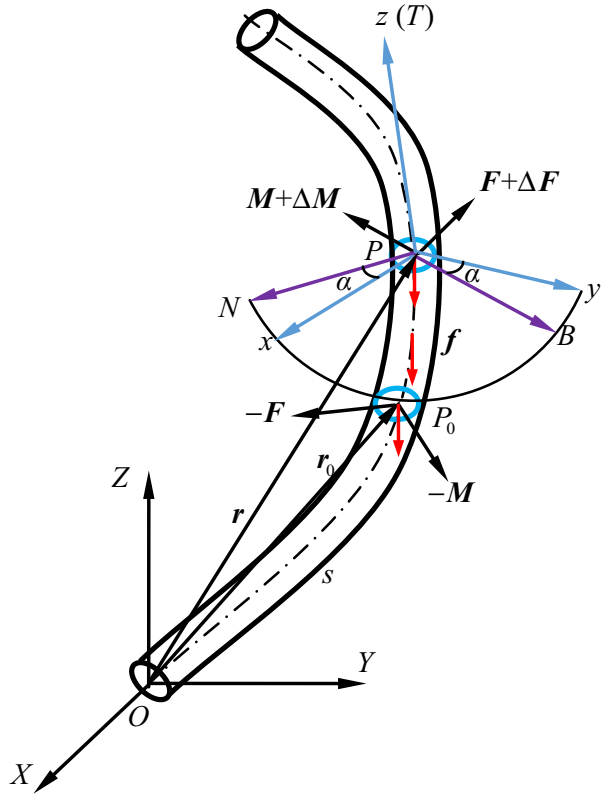

Fig. 3 Coordinate system transformation for the cable model

\section{Coordinate system $P-x y z$}

The coordinate system $P-x y z$ is the spindle coordinate system for the cable section (cable section and coordinate system $P-x y z$ are fixed). The $z$-axis coincides with the $T$-axis, while the plane $N P B$ coincides with the plane $x P y$. The angle between the $N$ - and $x$-axes is equal to that between the $B$ - and $y$-axes, and is denoted as $\alpha$, which represents the twist angle. The twist angle $\alpha$ reveals the torsional characteristics of the cable. The use of the transformation matrix $\boldsymbol{D}$ can overcome the pose problem between the $P-N B T$ and $P-x y z$ coordinate systems:

$\left[\begin{array}{l}x \\ y \\ z\end{array}\right]=\boldsymbol{D}\left[\begin{array}{l}N \\ B \\ T\end{array}\right]$
$\boldsymbol{D}=\left[\begin{array}{ccc}\cos \alpha & \sin \alpha & 0 \\ -\sin \alpha & \cos \alpha & 0 \\ 0 & 0 & 1\end{array}\right]$

Using the curvature $\kappa$, and torsion $\tau$ and twist angle $\alpha$ occurring at the point $P$, we define the curvaturetwisting vector $\omega$ of the point $P$ as

$$
\begin{aligned}
& \omega_{x}=\kappa \sin \alpha \\
& \omega_{y}=\kappa \cos \alpha \\
& \omega_{z}=\tau+\mathrm{d} \alpha / \mathrm{d} s
\end{aligned}
$$


where $\left(\omega_{x}, \omega_{y}, \omega_{z}\right)$ is the curvature-twisting vector $\boldsymbol{\omega}$ projected onto the coordinate system $P-x y z$.

\subsection{Static equilibrium equations for the cable}

Taking into account that the motion speed of the robot is relatively small and stable, and that $\theta_{j 1}<1^{\circ}$, we can statically analyze the cable; this means that we can ignore the dynamical processes.

As is shown in Fig. 3, in the world coordinate system $O-X Y Z$, the microarc $P_{0} P$ of the cable is considered in the static analysis. A gravitational force per unit length $f$ acts on the microarc $P_{0} P$, which is oriented opposite to the $Z$-axis. Assuming that the arc coordinate of the point $P_{0}$ is $s_{0}$, the arc coordinate of the point $P$ is $s$, with $s=s_{0}+\Delta s$ and $\Delta s>0$. When the microarc $P_{0} P$ is in equilibrium, the torque generated by the gravitational force per unit length $f$ is the second-order small value of $\Delta s$, which is negligible because the shape of the cross section of the cable is round. Thus, based on Yanzhu Liu's study [22], we obtain the following:

$\Delta \boldsymbol{F}+\boldsymbol{f} \Delta s=\mathbf{0}$

$\Delta \boldsymbol{M}+\Delta \boldsymbol{r} \times \boldsymbol{F}=\mathbf{0}$

Then, we divide Eqs. (6) and (7) by $\Delta s(\Delta s \rightarrow 0)$. Therefore,

$$
\begin{aligned}
& \frac{\mathrm{d} \boldsymbol{F}}{\mathrm{d} s}+\boldsymbol{f}=\mathbf{0} \\
& \frac{\mathrm{d} \boldsymbol{M}}{\mathrm{d} s}+\boldsymbol{T} \times \boldsymbol{F}=\mathbf{0}
\end{aligned}
$$

By changing the reference coordinate system for Eqs. (8) and (9) to the coordinate system $P-x y z$, we obtain

$$
\begin{aligned}
& \frac{\tilde{\mathrm{d}} \boldsymbol{F}}{\mathrm{d} s}+\boldsymbol{\omega} \times \boldsymbol{F}+\boldsymbol{f}=\mathbf{0} \\
& \frac{\tilde{\mathrm{d}} \boldsymbol{M}}{\mathrm{d} s}+\boldsymbol{\omega} \times \boldsymbol{M}+\boldsymbol{T} \times \boldsymbol{F}=\mathbf{0}
\end{aligned}
$$

Equations (10) and (11) are projected onto the coordinate system $P-x y z$. Thus, we obtain

$$
\begin{aligned}
& \frac{\mathrm{d} F_{x}}{\mathrm{~d} s}+\omega_{y} F_{z}-\omega_{z} F_{y}+f_{x}=0 \\
& \frac{\mathrm{d} F_{y}}{\mathrm{~d} s}+\omega_{z} F_{x}-\omega_{x} F_{z}+f_{y}=0 \\
& \frac{\mathrm{d} F_{z}}{\mathrm{~d} s}+\omega_{x} F_{y}-\omega_{y} F_{x}+f_{z}=0
\end{aligned}
$$

$$
\begin{aligned}
& \frac{\mathrm{d} M_{x}}{\mathrm{~d} s}+\omega_{y} M_{z}-\omega_{z} M_{y}-F_{y}=0 \\
& \frac{\mathrm{d} M_{y}}{\mathrm{~d} s}+\omega_{z} M_{x}-\omega_{x} M_{z}+F_{x}=0 \\
& \frac{\mathrm{d} M_{z}}{\mathrm{~d} s}+\omega_{x} M_{y}-\omega_{y} M_{x}=0
\end{aligned}
$$

The wavy lines in Eqs. (10) and (11) indicate that the reference coordinate system is $P-x y z$, which is different from that employed for Eqs. (8) and (9). Assuming that the cable has no initial curvature or twist, and that the constitutive relationship of the cable is known, we obtain

$$
\begin{aligned}
M_{x} & =A \omega_{x} \\
M_{y} & =B \omega_{y} \\
M_{z} & =C \omega_{z}
\end{aligned}
$$

where $A$ and $B$ represent the flexural stiffnesses about the $x$ - and $y$-axes of the cable cross section, respectively, and $C$ is the torsional stiffness of the cable cross section about the $z$-axis.

$$
\begin{aligned}
& A=E I_{x} \\
& B=E I_{y} \\
& C=G I_{z}
\end{aligned}
$$

Assuming that the cable is uniform and isotropic, $E$ and $G$ represent the Young's modulus and shear modulus of the cable, respectively. $I_{x}$ and $I_{y}$ are the moments of inertia for the cable cross section with respect to the $x$ - and $y$-axes, and $I_{z}$ is the polar moment of inertia for the cable cross section with respect to the $z$-axis.

For a cable with a diameter $d_{\mathrm{c}}$,

$$
\begin{aligned}
& I_{x}=I_{y}=\frac{\pi d_{\mathrm{c}}^{4}}{64} \\
& I_{z}=\frac{\pi d_{\mathrm{c}}^{4}}{32}
\end{aligned}
$$

As is shown in Fig. $4, q_{k}(k=1,2,3,4)$ are the Euler parameters for the cable cross section (the cable cross section is perpendicular to the $z$-axis.). The Euler parameters can be determined by Eq. (17). The coordinate system $P-x y z$ is rotated about the axis $\boldsymbol{e}$ by an angle $\phi$ with respect to the coordinate system $O-X Y Z$. 


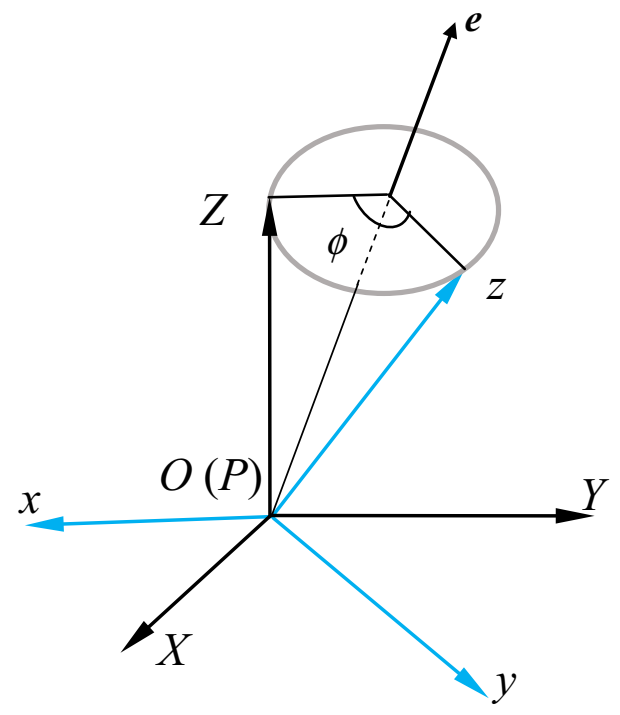

Fig. 4 Geometric interpretation of the Euler parameters

$$
\begin{aligned}
& q_{1}=\cos \frac{\phi}{2} \\
& q_{k+1}=e_{k} \sin \frac{\phi}{2}(k=1,2,3)
\end{aligned}
$$

where $e_{k}(k=1,2,3)$ correspond to the direction cosines of the limited axis of rotation $\boldsymbol{e}$ with respect to the world coordinate system $O-X Y Z ; \phi$ is the limited rotation angle.

The derivatives of $q_{k}(k=1,2,3,4)$ with respect to the arc coordinate $s$ are employed as new variables, as follows:

$Q_{k}=\frac{\mathrm{d} q_{k}}{\mathrm{~d} s}(k=1,2,3,4)$

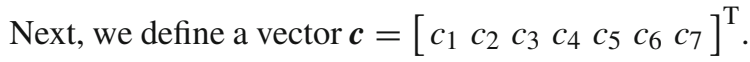

$c_{1}=q_{1}^{2}+q_{2}^{2}+q_{3}^{2}+q_{4}^{2}-1$

According to the infinitesimally small rotation theory of a rigid body [22,32], [33, (Page 16)],

$$
\begin{aligned}
& \omega_{x}=2\left(-q_{2} Q_{1}+q_{1} Q_{2}+q_{4} Q_{3}-q_{3} Q_{4}\right) \\
& \omega_{y}=2\left(-q_{3} Q_{1}-q_{4} Q_{2}+q_{1} Q_{3}+q_{2} Q_{4}\right) \\
& \omega_{z}=2\left(-q_{4} Q_{1}+q_{3} Q_{2}-q_{2} Q_{3}+q_{1} Q_{4}\right)
\end{aligned}
$$

If Eq. (20) is substituted into Eqs. (12) and (13), we obtain [22]:

$$
\begin{aligned}
c_{2}= & \frac{\mathrm{d} F_{x}}{\mathrm{~d} s}+2\left(V_{2} F_{z}-V_{3} F_{y}\right)+f_{x} \\
c_{3}= & \frac{\mathrm{d} F_{y}}{\mathrm{~d} s}+2\left(V_{3} F_{x}-V_{1} F_{z}\right)+f_{y} \\
c_{4}= & \frac{\mathrm{d} F_{z}}{\mathrm{~d} s}+2\left(V_{1} F_{y}-V_{2} F_{x}\right)+f_{z} \\
c_{5}= & -q_{2} \frac{\mathrm{d} Q_{1}}{\mathrm{~d} s}+q_{1} \frac{\mathrm{d} Q_{2}}{\mathrm{~d} s}+q_{4} \frac{\mathrm{d} Q_{3}}{\mathrm{~d} s}-q_{3} \frac{\mathrm{d} Q_{4}}{\mathrm{~d} s}-\frac{F_{y}}{2 A}+\frac{2(C-B)}{A} V_{2} V_{3} \\
c_{6}= & -q_{3} \frac{\mathrm{d} Q_{1}}{\mathrm{~d} s}-q_{4} \frac{\mathrm{d} Q_{2}}{\mathrm{~d} s}+q_{1} \frac{\mathrm{d} Q_{3}}{\mathrm{~d} s}+q_{2} \frac{\mathrm{d} Q_{4}}{\mathrm{~d} s}+\frac{F_{x}}{2 B}+\frac{2(A-C)}{B} V_{3} V_{1} \\
c_{7}= & -q_{4} \frac{\mathrm{d} Q_{1}}{\mathrm{~d} s}+q_{3} \frac{\mathrm{d} Q_{2}}{\mathrm{~d} s}-q_{2} \frac{\mathrm{d} Q_{3}}{\mathrm{~d} s}+q_{1} \frac{\mathrm{d} Q_{4}}{\mathrm{~d} s} \\
& +\frac{2(B-A)}{C} V_{1} V_{2}
\end{aligned}
$$

where

$$
\begin{aligned}
& V_{1}=-q_{2} Q_{1}+q_{1} Q_{2}+q_{4} Q_{3}-q_{3} Q_{4} \\
& V_{2}=-q_{3} Q_{1}-q_{4} Q_{2}+q_{1} Q_{3}+q_{2} Q_{4} \\
& V_{3}=-q_{4} Q_{1}+q_{3} Q_{2}-q_{2} Q_{3}+q_{1} Q_{4}
\end{aligned}
$$

When the cable is in equilibrium, we have the following:

$\boldsymbol{c}=\left[\begin{array}{lllllll}c_{1} & c_{2} & c_{3} & c_{4} & c_{5} & c_{6} & c_{7}\end{array}\right]^{\mathrm{T}}=\mathbf{0}$

\section{Deriving the solution}

\subsection{Discretization of the equations}

Equation (22) can be solved by using the finite difference method. We assume that the length of the cable before and after deformation is the same $\left(L_{0}\right)$ (Fig. 5). The cable is uniformly divided into $n$ parts with lengths of $\tilde{l}_{i}=\tilde{l}_{0}=L_{0} / n$. Therefore, the cable has $(n+1)$ discrete points and $n$ calculation units. The calculation unit between the discrete points $P_{i}$ and $P_{i+1}$ is denoted as Part $i(1 \leq i \leq n)$.

When $n \rightarrow \infty$,

$\mathrm{d} s=\Delta s=\tilde{l}_{i}$

All of the calculations performed in this study are derived as based on the assumption that Eq. (23) is valid. The Euler parameters for the discrete points $P_{i}$ and $P_{i+1}$ are $q_{k, i}$ and $q_{k, i+1}(k=1,2,3,4)$, the internal forces are $\left(F_{x, i}, F_{y, i}, F_{z, i}\right) \quad$ and $\left(F_{x, i+1}, F_{y, i+1}, F_{z, i+1}\right)$, the change rates of the Euler 


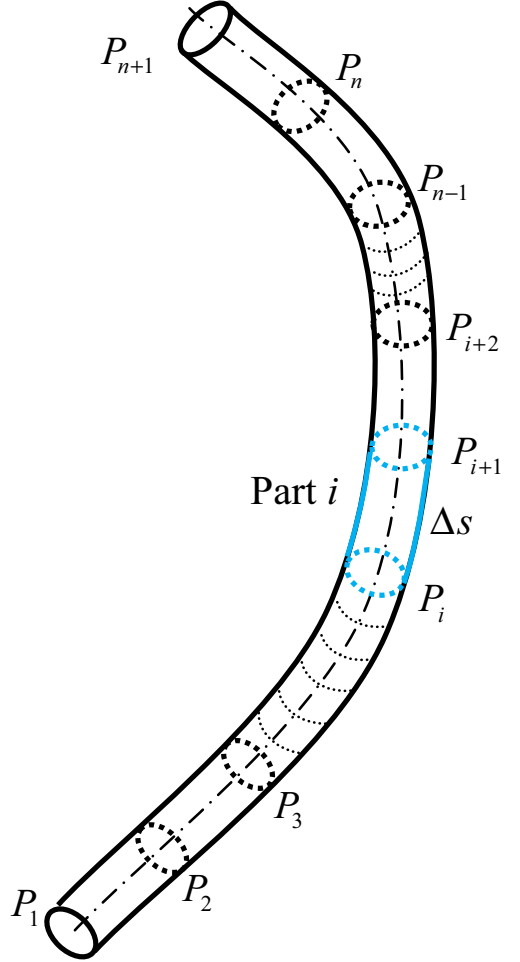

Fig. 5 Discretization of the cable

parameters are $Q_{k, i}$ and $Q_{k, i+1}(k=1,2,3,4)$, and the change rates of the internal forces are $\left(\frac{\mathrm{d} F_{x, i}}{\mathrm{~d} s}\right.$, $\left.\frac{\mathrm{d} F_{y, i}}{\mathrm{~d} s}, \frac{\mathrm{d} F_{z, i}}{\mathrm{~d} s}\right)$ and $\left(\frac{\mathrm{d} F_{x, i+1}}{\mathrm{~d} s}, \frac{\mathrm{d} F_{y, i+1}}{\mathrm{~d} s}, \frac{\mathrm{d} F_{z, i+1}}{\mathrm{~d} s}\right)$, respectively.

For Part $i$ ( $i$ th calculation unit),

$$
\begin{aligned}
& Q_{k, i+1} \\
& =\left\{\begin{array}{l}
\frac{-3 q_{k, 1}+4 q_{k, 2}-q_{k, 3}}{2 \tilde{l}_{0}} i=0 \\
\frac{q_{k, i+1}-q_{k, i}}{\tilde{l}_{0}} 1 \leq i \leq n
\end{array} \quad(k=1,2,3,4)\right. \\
& J_{k, i}=\frac{\mathrm{d} Q_{k, i+1}}{\mathrm{~d} s}=\frac{Q_{k, i+1}-Q_{k, i}}{\tilde{l}_{0}} \\
& \frac{\mathrm{d} F_{x, i+1}}{\mathrm{~d} s}=\frac{F_{x, i+1}-F_{x, i}}{\tilde{l}_{0}} \\
& \frac{\mathrm{d} F_{y, i+1}}{\mathrm{~d} s}=\frac{F_{y, i+1}-F_{y, i}}{\tilde{l}_{0}} \\
& \frac{\mathrm{d} F_{z, i+1}}{\mathrm{~d} s}=\frac{F_{z, i+1}-F_{z, i}}{\tilde{l}_{0}}
\end{aligned}
$$

Using Eq. (22), we determine that, for each calcula-

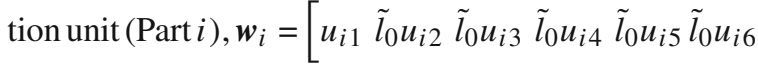
$\left.\tilde{l}_{0} u_{i 7}\right]^{\mathrm{T}}$. Therefore,

$$
\begin{aligned}
& u_{i 1}=q_{1, i+1}^{2}+q_{2, i+1}^{2}+q_{3, i+1}^{2}+q_{4, i+1}^{2}-1 \\
& u_{i 2}=\frac{F_{x, i+1}-F_{x, i}}{\tilde{l}_{0}}+2\left(V_{2, i} F_{z, i+1}-V_{3, i} F_{y, i+1}\right)+f_{x, i+1} \\
& u_{i 3}=\frac{F_{y, i+1}-F_{y, i}}{\tilde{l}_{0}}+2\left(V_{3, i} F_{x, i+1}-V_{1, i} F_{z, i+1}\right)+f_{y, i+1} \\
& u_{i 4}=\frac{F_{z, i+1}-F_{z, i}}{\tilde{l}_{0}}+2\left(V_{1, i} F_{y, i+1}-V_{2, i} F_{x, i+1}\right)+f_{z, i+1}
\end{aligned}
$$

$$
\begin{aligned}
u_{i 5}= & -q_{2, i+1} J_{1, i}+q_{1, i+1} J_{2, i}+q_{4, i+1} J_{3, i} \\
& -q_{3, i+1} J_{4, i}+\frac{2(C-B)}{A} V_{2, i} V_{3, i}-\frac{F_{y, i+1}}{2 A} \\
u_{i 6}= & -q_{3, i+1} J_{1, i}-q_{4, i+1} J_{2, i}+q_{1, i+1} J_{3, i} \\
& +q_{2, i+1} J_{4, i}+\frac{2(A-C)}{B} V_{3, i} V_{1, i}+\frac{F_{x, i+1}}{2 B} \\
u_{i 7}= & -q_{4, i+1} J_{1, i}+q_{3, i+1} J_{2, i}-q_{2, i+1} J_{3, i} \\
& +q_{1, i+1} J_{4, i}+\frac{2(B-A)}{C} V_{1, i} V_{2, i}
\end{aligned}
$$

where

$$
\begin{aligned}
V_{1, i}= & -q_{2, i+1} Q_{1, i+1}+q_{1, i+1} Q_{2, i+1} \\
& +q_{4, i+1} Q_{3, i+1}-q_{3, i+1} Q_{4, i+1} \\
V_{2, i}= & -q_{3, i+1} Q_{1, i+1}-q_{4, i+1} Q_{2, i+1} \\
& +q_{1, i+1} Q_{3, i+1}+q_{2, i+1} Q_{4, i+1} \\
V_{3, i}= & -q_{4, i+1} Q_{1, i+1}+q_{3, i+1} Q_{2, i+1} \\
& -q_{2, i+1} Q_{3, i+1}+q_{1, i+1} Q_{4, i+1}
\end{aligned}
$$

Next, we define a vector $\boldsymbol{u}=$ $\left[\boldsymbol{w}_{1}^{\mathrm{T}} \boldsymbol{w}_{2}^{T} \cdots \boldsymbol{w}_{i}^{\mathrm{T}} \cdots \boldsymbol{w}_{n-1}^{\mathrm{T}} \boldsymbol{w}_{n}^{\mathrm{T}}\right]^{\mathrm{T}}$. When the cable is in equilibrium, we obtain

$$
\boldsymbol{u}=\left[\boldsymbol{w}_{1}^{\mathrm{T}} \boldsymbol{w}_{2}^{\mathrm{T}} \cdots \boldsymbol{w}_{i}^{\mathrm{T}} \cdots \boldsymbol{w}_{n-1}^{\mathrm{T}} \boldsymbol{w}_{n}^{\mathrm{T}}\right]^{\mathrm{T}}=\mathbf{0}
$$


The rotation matrix of the coordinate system $P_{i+1}$ $-x y z$ at the discrete point $P_{i+1}$, with respect to the world coordinate system $O-X Y Z$, is

$$
\begin{aligned}
& -\left(2 q_{1, j-1}+q_{1, j}\right) q_{2, j-1} \\
& \left.-\left(2 q_{1, j}+q_{1, j-1}\right) q_{2, j}\right]
\end{aligned}
$$

${ }_{i+1}^{P_{i+1}} \boldsymbol{R}=\left[\begin{array}{lll}q_{1, i+1}^{2}+q_{2, i+1}^{2}-q_{3, i+1}^{2}-q_{4, i+1}^{2} & 2\left(q_{2, i+1} q_{3, i+1}-q_{1, i+1} q_{4, i+1}\right) & 2\left(q_{2, i+1} q_{4, i+1}+q_{1, i+1} q_{3, i+1}\right) \\ 2\left(q_{2, i+1} q_{3, i+1}+q_{1, i+1} q_{4, i+1}\right) & q_{1, i+1}^{2}-q_{2, i+1}^{2}+q_{3, i+1}^{2}-q_{4, i+1}^{2} & 2\left(q_{3, i+1} q_{4, i+1}-q_{1, i+1} q_{2, i+1}\right) \\ 2\left(q_{2, i+1} q_{4, i+1}-q_{1, i+1} q_{3, i+1}\right) & 2\left(q_{3, i+1} q_{4, i+1}+q_{1, i+1} q_{2, i+1}\right) & q_{1, i+1}^{2}-q_{2, i+1}^{2}-q_{3, i+1}^{2}+q_{4, i+1}^{2}\end{array}\right]$

The rotation matrix ${ }_{O}^{P_{i+1}} \boldsymbol{R}$ is an orthogonal matrix, i.e., its inverse matrix is equal to its transposed matrix.

$$
{ }_{O}^{P_{i+1}} \boldsymbol{R}^{-1}={ }_{O}^{P_{i+1}} \boldsymbol{R}^{\mathrm{T}}
$$

The distribution forces on the calculation unit Part $i$ are concentrated at the discrete point $P_{i+1}$. The gravitational force per unit length $f$ at the discrete point $P_{i+1}$ is projected as $\left(0,0, f_{0}\right)$ in the world coordinate system $O-X Y Z$. The projection of $f$ onto the coordinate system $P_{i+1}-x y z$ is:

$$
\left[\begin{array}{l}
f_{x, i+1} \\
f_{y, i+1} \\
f_{z, i+1}
\end{array}\right]=\stackrel{P_{i+1}}{O} \boldsymbol{R}^{-1}\left[\begin{array}{l}
0 \\
0 \\
f_{0}
\end{array}\right]
$$

If we substitute Eq. (30) into Eqs. (32), (33) is obtained.

$$
\left[\begin{array}{l}
f_{x, i+1} \\
f_{y, i+1} \\
f_{z, i+1}
\end{array}\right]=f_{0}\left[\begin{array}{l}
2\left(q_{2, i+1} q_{4, i+1}-q_{1, i+1} q_{3, i+1}\right) \\
2\left(q_{3, i+1} q_{4, i+1}+q_{1, i+1} q_{2, i+1}\right) \\
q_{1, i+1}^{2}-q_{2, i+1}^{2}-q_{3, i+1}^{2}+q_{4, i+1}^{2}
\end{array}\right]
$$

The point $P_{1}$ coincides with the origin $O$ of the world coordinate system. In the $O-X Y Z$ coordinate system, $\boldsymbol{P}_{1} \boldsymbol{P}_{i+1}=\boldsymbol{r}_{i+1}=\left(r_{X, i+1}, r_{Y, i+1}, r_{Z, i+1}\right)$, and the value of $\boldsymbol{P}_{1} \boldsymbol{P}_{n+1}=\left(r_{X}, r_{Y}, r_{Z}\right)$ should be known. Using Eqs. (2) and (30), we obtain the following [22, 23, 25]:

$$
\begin{aligned}
r_{X, i+1}= & \frac{1}{3} \sum_{j=2}^{i+1} \tilde{l}_{0}\left[\left(2 q_{2, j-1}+q_{2, j}\right) q_{4, j-1}\right. \\
& +\left(2 q_{2, j}+q_{2, j-1}\right) q_{4, j} \\
& +\left(2 q_{1, j-1}+q_{1, j}\right) q_{3, j-1} \\
& \left.+\left(2 q_{1, j}+q_{1, j-1}\right) q_{3, j}\right] \\
r_{Y, i+1}= & \frac{1}{3} \sum_{j=2}^{i+1} \tilde{l}_{0}\left[\left(2 q_{3, j-1}+q_{3, j}\right) q_{4, j-1}\right. \\
& +\left(2 q_{3, j}+q_{3, j-1}\right) q_{4, j}
\end{aligned}
$$

$$
\begin{aligned}
r_{Z, i+1}= & \frac{1}{3} \sum_{j=2}^{i+1} \tilde{l}_{0}\left[2 \left(q_{1, j-1}^{2}+q_{1, j-1} q_{1, j}+q_{1, j}^{2}\right.\right. \\
& \left.\left.+q_{4, j-1}^{2}+q_{4, j-1} q_{4, j}+q_{4, j}^{2}\right)-3\right] \\
r_{X, n+1}= & r_{X} \\
r_{Y, n+1}= & r_{Y} \\
r_{Z, n+1}= & r_{Z}
\end{aligned}
$$

By implementing the cable length condition [23-25], we obtain

$c_{0}=\sum_{i=1}^{n} \sqrt{\left(r_{X, i+1}-r_{X, i}\right)^{2}+\left(r_{Y, i+1}-r_{Y, i}\right)^{2}+\left(r_{Z, i+1}-r_{Z, i}\right)^{2}}-L_{0}=0$

The boundary conditions of the cable (Euler parameters of the discrete points $P_{1}$ and $P_{n+1}$ ) are

$$
\left\{\begin{array} { l } 
{ q _ { 1 , 1 } = a _ { 1 } } \\
{ q _ { 2 , 1 } = a _ { 2 } } \\
{ q _ { 3 , 1 } = a _ { 3 } } \\
{ q _ { 4 , 1 } = a _ { 4 } } \\
{ a _ { 1 } ^ { 2 } + a _ { 2 } ^ { 2 } + a _ { 3 } ^ { 2 } + a _ { 4 } ^ { 2 } = 1 }
\end{array} \quad \left\{\begin{array}{l}
q_{1, n+1}=b_{1} \\
q_{2, n+1}=b_{2} \\
q_{3, n+1}=b_{3} \\
q_{4, n+1}=b_{4} \\
b_{1}^{2}+b_{2}^{2}+b_{3}^{2}+b_{4}^{2}=1
\end{array}\right.\right.
$$

$u_{n 1}$ corresponds to the calculation unit Part $n$, and the squared sum of the Euler parameters at the discrete point $P_{n+1}$ is repeatedly defined; hence, $u_{n 1}$ is replaced by $c_{0}$ in Eq. (39). If the cable is in equilibrium, Eq. (39) is valid. 


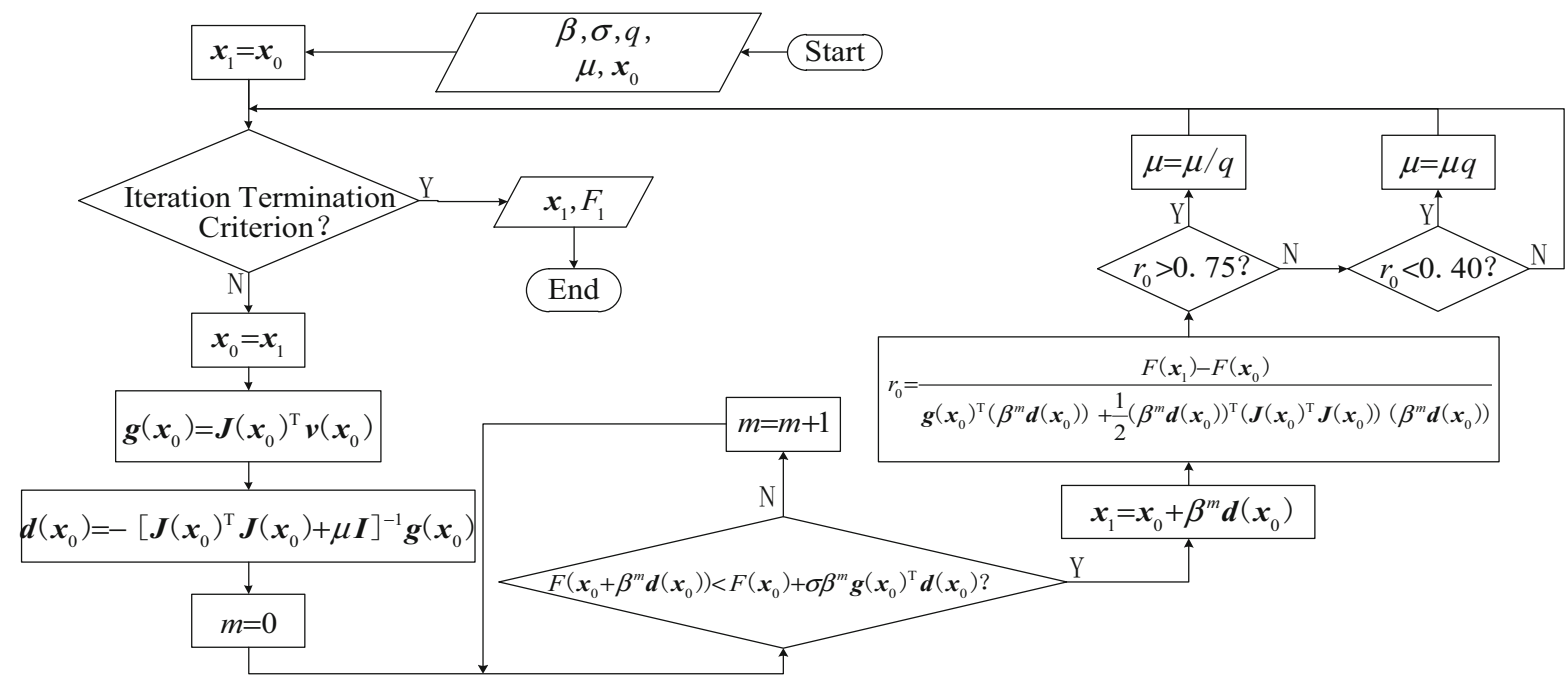

Fig. 6 Flowchart for the numerical iterative algorithm

$\boldsymbol{v}=\left[\begin{array}{c}r_{X}-r_{X, n+1} \\ r_{Y}-r_{Y, n+1} \\ r_{Z}-r_{Z, n+1} \\ q_{1,1}-a_{1} \\ q_{2,1}-a_{2} \\ q_{3,1}-a_{3} \\ q_{4,1}-a_{4} \\ q_{1, n+1}-b_{1} \\ q_{2, n+1}-b_{2} \\ q_{3, n+1}-b_{3} \\ q_{4, n+1}-b_{4} \\ \boldsymbol{u}\end{array}\right]=\mathbf{0}$

Equation (39) determines the parameters for all discrete points in Eq. (40).

$$
\begin{aligned}
& x_{k, i}=q_{k, i}(k=1,2,3,4,1 \leq i \leq n+1) \\
& \left(x_{5, i}, x_{6, i}, x_{7, i}\right)=\left(F_{x, i}, F_{y, i}, F_{z, i}\right)
\end{aligned}
$$

\subsection{Numerical iteration calculations}

Equation (39) can be regarded as a nonlinear leastsquares problem that equates to the global optimization problem $[\min F(\boldsymbol{x})]$.

$\min F(\boldsymbol{x})=\frac{1}{2} \min \sum_{j=1}^{7 n+11} v_{j}^{2}$

In an ideal case, the optimal solution of the problem is determined by $\min F(\boldsymbol{x})=0$, where $\boldsymbol{x}$ is a $7(n+1)$ dimensional vector, $\boldsymbol{x}_{0}$ represents the iteration initial value [Eq. (41)], and $\boldsymbol{x}_{k}$ represents the $k^{\text {th }}$ iteration point. The output value of $F(\boldsymbol{x})$ is compared to the numerical iteration termination criterion for each iteration.

In [24], the Levenberg-Marquardt algorithm is proposed to solve the cable model under the condition of constraints imposed at both ends; however, this algorithm may cannot guarantee numerical convergence of the cable model under complex conditions. Thus, in this study, the algorithm is improved by using the trustregion method to ensure that the theoretical model of the cable in contact with a curved surface has good numerical stability. A flowchart for the numerical iterative algorithm is presented in Fig. 6.

The convergence of the calculation results is influenced by the iterative initial value, and the iterative initial value $\boldsymbol{x}_{0}$ is selected as a column vector whose most components are 1. Define $\boldsymbol{g}\left(\boldsymbol{x}_{k}\right)$ as the gradient of the function $F(\boldsymbol{x})$ at $\boldsymbol{x}_{k}, \boldsymbol{J}\left(\boldsymbol{x}_{k}\right)$ is the Jacobian matrix for $\boldsymbol{v}$ at $\boldsymbol{x}_{k}, \boldsymbol{I}$ is the unit matrix, $\boldsymbol{d}_{k}$ is the iterative step of $\boldsymbol{x}_{k}$, and $\mu_{k}$ is the adjustment parameter for the iteration step $\boldsymbol{d}_{k}$. Therefore,

$$
\begin{aligned}
& \boldsymbol{g}\left(\boldsymbol{x}_{k}\right)=\nabla F\left(\boldsymbol{x}_{k}\right)=\boldsymbol{J}\left(\boldsymbol{x}_{k}\right)^{T} \boldsymbol{v}\left(\boldsymbol{x}_{k}\right) \\
& \boldsymbol{d}_{k}=-\left[\boldsymbol{J}\left(\boldsymbol{x}_{k}\right)^{T} \boldsymbol{J}\left(\boldsymbol{x}_{k}\right)+\mu_{k} \boldsymbol{I}\right]^{-1} \boldsymbol{g}\left(\boldsymbol{x}_{k}\right)
\end{aligned}
$$

According to Eqs. (44) and (45), the parameter $\mu_{k}$ is adjusted by using the trust-region method; $r_{k}$ is the radius of the trust region of the parameter $\mu_{k}, q$ is the scaling factor of the parameter $\mu_{k}$, where $q=2 \sim 10$. The critical values for $r_{k}$ and scaling factor $q$ can be, 
Fig. 7 Theoretical model of the 2-DOF space observation robotic arm

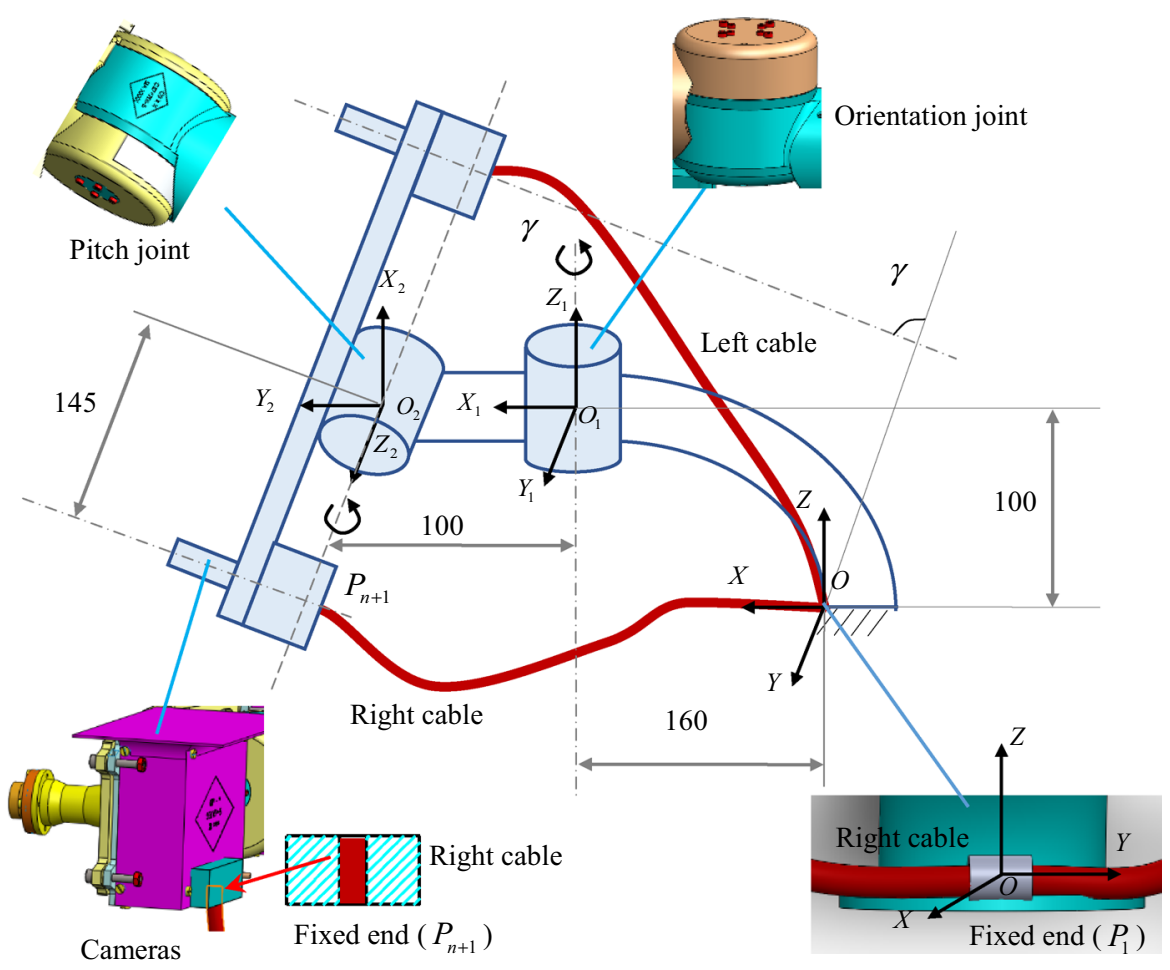

respectively, adjusted according to the actual situation. Thus, we obtain

$r_{k}=\frac{F\left(\boldsymbol{x}_{k+1}\right)-F\left(\boldsymbol{x}_{k}\right)}{\boldsymbol{g}\left(\boldsymbol{x}_{k}\right)^{T}\left(\beta^{m} \boldsymbol{d}\left(\boldsymbol{x}_{k}\right)\right)+\frac{1}{2}\left(\beta^{m} \boldsymbol{d}\left(\boldsymbol{x}_{k}\right)\right)^{T}\left(\boldsymbol{J}\left(\boldsymbol{x}_{k}\right)^{T} \boldsymbol{J}\left(\boldsymbol{x}_{k}\right)\right)\left(\beta^{m} \boldsymbol{d}\left(\boldsymbol{x}_{k}\right)\right)}$

$\mu_{k}=\left\{\begin{array}{lc}q \mu_{k} & r_{k}<0.4 \\ \mu_{k} & 0.4 \leq r_{k} \leq 0.75 \\ \mu_{k} / q & r_{k}>0.75\end{array}\right.$

The step size factor for the iterative calculations is obtained by using the Armijo criterion, where $\beta \in$ $(0,1)$ and $\sigma \in(0,0.5)$.

$F\left(\boldsymbol{x}_{k}+\beta^{m} \boldsymbol{d}\left(\boldsymbol{x}_{k}\right)\right) \leq F\left(\boldsymbol{x}_{k}\right)+\sigma \beta^{m} \boldsymbol{g}\left(\boldsymbol{x}_{k}\right)^{T} \boldsymbol{d}\left(\boldsymbol{x}_{k}\right)$

\subsection{Calculation results}

The Denavit-Hartenberg coordinate systems for the 2DOF observation robot are shown in Fig. 7 (unit of length: mm). The robot has two DOFs; it has an orientation joint and a pitch joint, which can realize the movement of the cameras carrying cables within a certain range. The origin of the world coordinate system $O-X Y Z$ is set to correspond to the starting point $O$ of the cable, and $O_{1}$ and $O_{2}$ are located at the geometric centers of the orientation and pitch joints, respectively. The experiment shows that the phenomenon of slow rotation may occur at the orientation joint, whereas it does not occur at the pitch joint. Therefore, in this study, a single joint, i.e., the orientation joint, is considered, while the pitch angle of the pitch joint is set to zero. For simplicity, only the right cable of the robot was employed for the analysis, and the experiments are currently being conducted using ordinary cables instead of real cables covered by the nonmetallic shielding layers. The $z$-axis direction of the coordinate system $P-x y z$ of the discrete point $P_{1}$ is opposite to the $Y$-axis direc-

Table 1 Material parameters of the cable

\begin{tabular}{lllll}
\hline Diameter $d_{\mathrm{c}}$ & Length $L_{0}$ & $\begin{array}{c}\text { Gravitational force } \\
\text { per unit length } f\end{array}$ & Young's modulus $E$ & Shear modulus $G$ \\
\hline $7.54 \mathrm{~mm}$ & $380 \mathrm{~mm}$ & $10^{-3} \mathrm{~N} / \mathrm{mm}$ & $6.70 \mathrm{MPa}$ & $2.28 \mathrm{MPa}$ \\
\hline
\end{tabular}


tion, and the $z$-axis direction at the discrete point $P_{n+1}$ is the same as the $Z$-axis. The $X$ - and $Z$-coordinates of the end point $P_{n+1}$ of the right cable are, respectively, 20 and $25 \mathrm{~mm}$ smaller than those of the point $O_{2}$ when $\gamma=0$. The coordinates of the point $P_{n+1}$ in the world coordinate system $O-X Y Z$ are

$\left\{\begin{array}{l}r_{X, n+1}=160-145 \sin \gamma+80 \cos \gamma \\ r_{Y, n+1}=80 \sin \gamma+145 \cos \gamma \\ r_{Z, n+1}=75\end{array} \quad\left(\gamma \in\left[-\frac{\pi}{2}, \frac{\pi}{2}\right]\right)\right.$

where $\gamma$ is the rotation angle of the orientation joint in the 2-DOF space observation robotic arm.

The material parameters of the cable are shown in Table 1. According to Eq. (39), the cable mechanical model considers the cable shape and mechanical properties. With the change in the rotation angle $\gamma$ of the orientation joint, the morphological curves of the robot cable are projected onto the $X O Z, Y O Z$, and $X O Y$ planes. Figure 8 shows the projection of the calculated trajectory of the robot cable onto each coordinate plane, and Fig. 9 shows the comparison between the theoretical shape of the robot cable and the actual trajectory. The trends of the cable theoretical shape and the actual trajectory are consistent. In addition, when the actual trajectory of the robot cable in the XOY plane is collected (by a camera sensor), the cable is blocked by the robotic arm. To obtain a more complete actual trajectory of the robot cable, the optical axis of a camera sensor used is not perpendicular to the $\mathrm{XOY}$ plane (there is a small angle of inclination), resulting in a slightly large error in the comparison of the cable shape in the $\mathrm{XOY}$ plane. Figure 10 shows the projection of the internal force onto the end of the cable in the $P-x y z$ coordinate system.

\section{Curved surface contact}

Taking into account the complexity of the contact between the cable and curved surface, in order to simplify the analysis, the diameter of the cable is ignored, and the surface is considered to be spherical. Therefore, a nonlinear model of the cable with spherical contact is developed. As is shown in Fig. 11, the coordinates of the center of the sphere $O_{0}$ are $\left(X_{0}, Y_{0}, Z_{0}\right)$, the radius is $R_{0}$, and the equation for the spherical surface $G$ is

$$
\begin{aligned}
F_{s p}(\boldsymbol{r})= & \sqrt{\left(X-X_{0}\right)^{2}+\left(Y-Y_{0}\right)^{2}+\left(Z-Z_{0}\right)^{2}} \\
& -R_{0}=0
\end{aligned}
$$

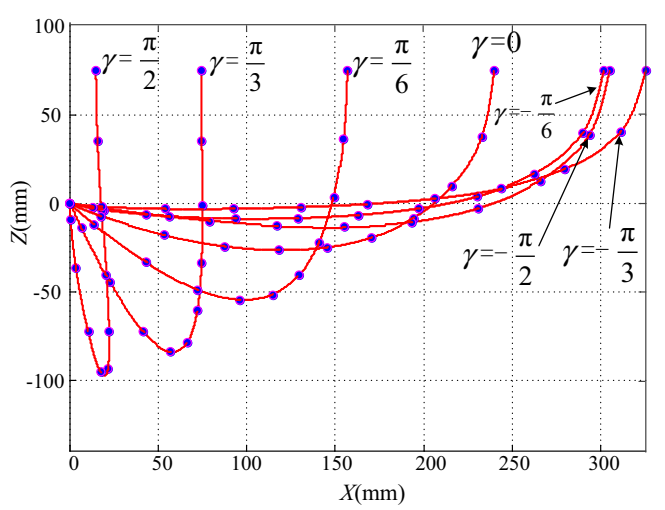

(a)

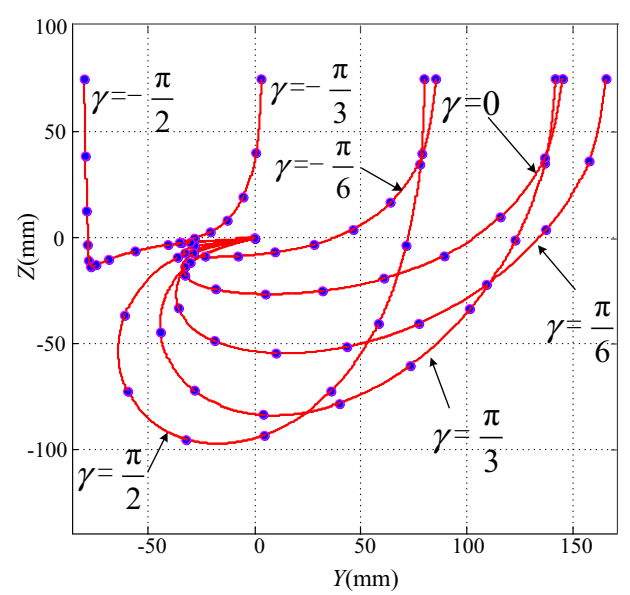

(b)

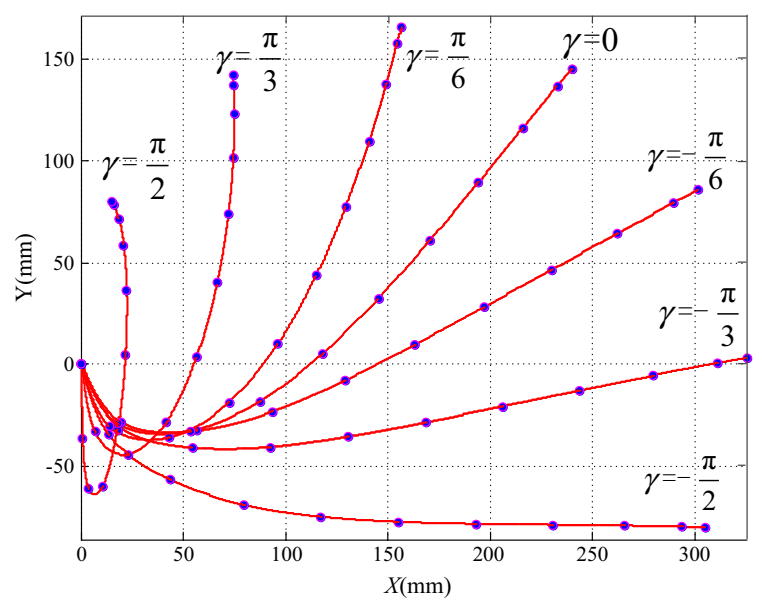

(c)

Fig. 8 Theoretical shape of the robot cable. Projections onto the a $X O Z$, b $Y O Z$, and $\mathbf{c} X O Y$ planes

For the actual solution, we first assume that the curved surface does not exist; by using Eq. (39), we can identify the coordinates of all discrete points. Then, we 
Fig. 9 Comparison of theoretical shape and actual trajectory of robot cable

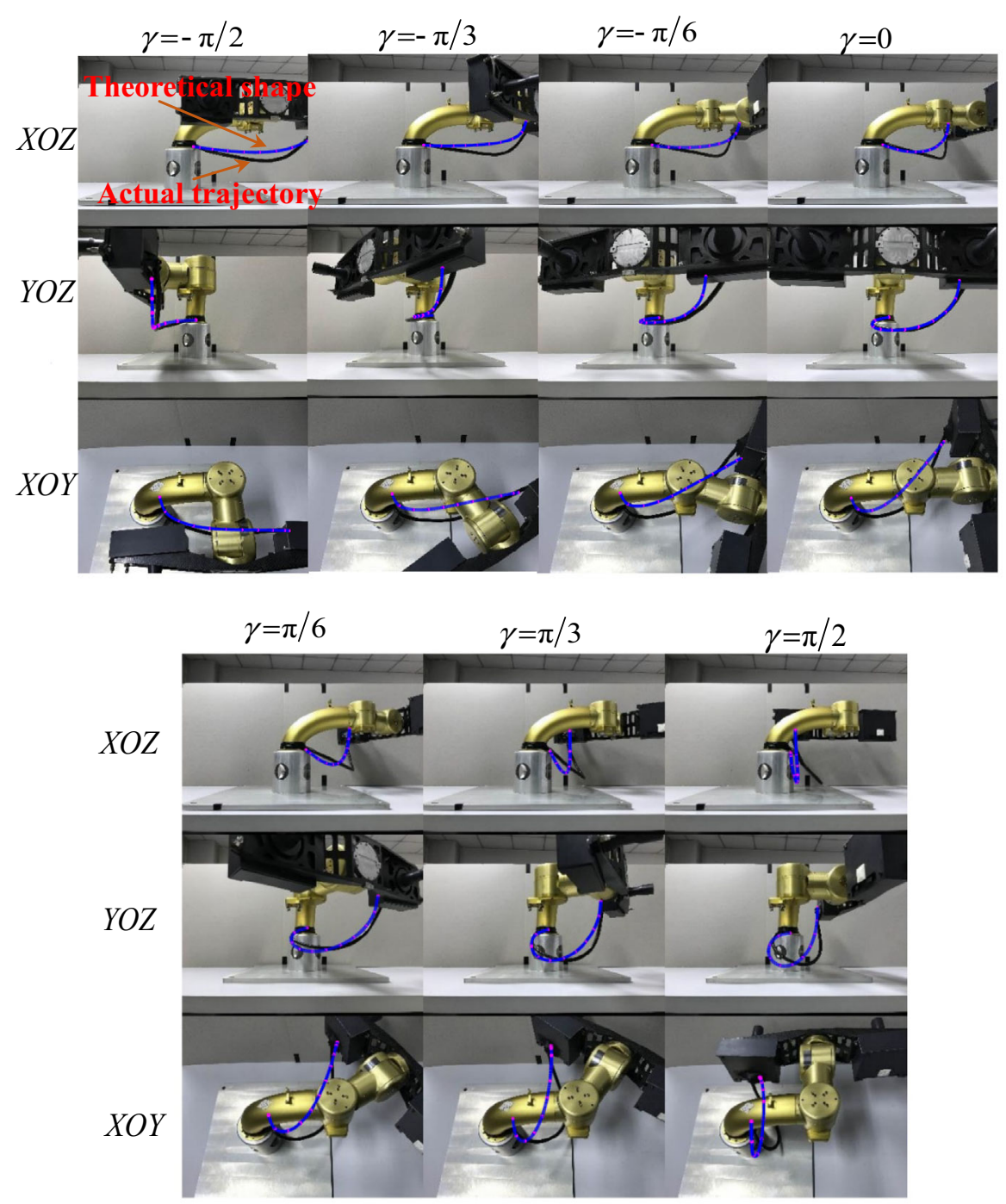

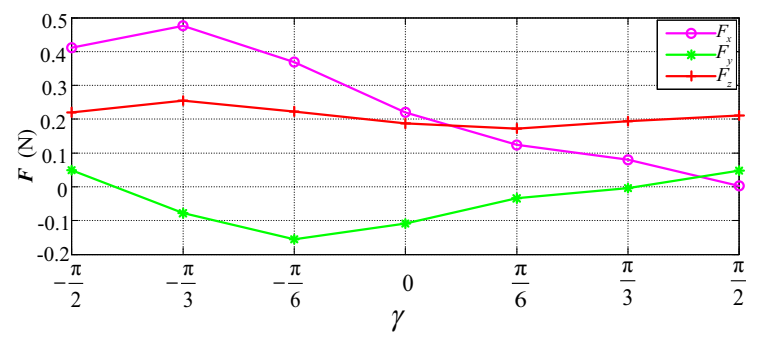

Fig. 10 End-point force on the robot cable

find all of the discrete points that satisfy Eq. (49) and use them as elements to generate the point set $U$.

$$
\begin{aligned}
& \sqrt{\left(r_{X, i+1}-X_{0}\right)^{2}+\left(r_{Y, i+1}-Y_{0}\right)^{2}+\left(r_{Z, i+1}-Z_{0}\right)^{2}} \\
& -R_{0}<0
\end{aligned}
$$

Next, let us assume that the number of elements in the point set $U$ is $n_{\mathrm{c}}\left(n_{\mathrm{c}} \geq 1\right)$. The subscript size $(i+1)$ of $P_{i+1} \in U$ is used as the standard for sorting. Then, we select the intermediate point $P_{d}$ in the point set $U$. If the number of cases is even, we choose a larger discrete subscript to ensure that there is a complete calculation unit in $U$. When the serial numbers of the cable discrete points in the curved surface are sorted from small to large, $t_{1}$ is the subscript size of the first discrete point. $d$ is the subscript size of the middle point of the cable discrete points in the curved surface. When $n_{\mathrm{c}}$ is an even number, $d=t_{1}+n_{\mathrm{c}} / 2$, and when $n_{\mathrm{c}}$ is an odd number, $d=t_{1}+\left(n_{\mathrm{c}}-1\right) / 2$.

Only the elements that belong to the point set $U$ are likely to be in contact with the curved surface. When 


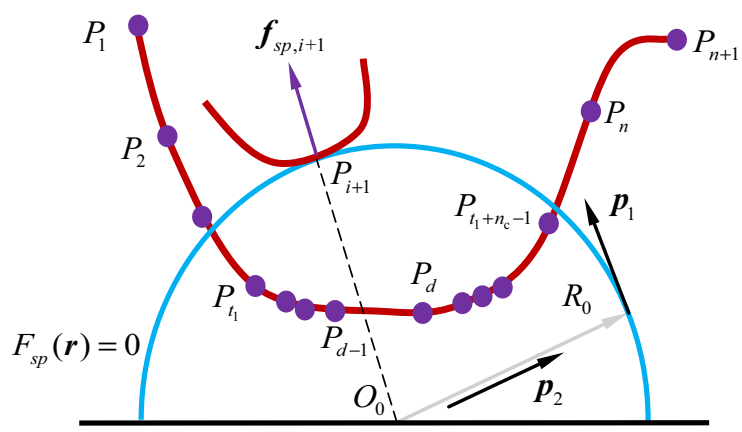

Fig. 11 Constraint of the cable which is in contact with a curved surface

the cable is in contact with the curved surface, the tangent unit vector $\boldsymbol{p}_{1}$ at the cable contact point is perpendicular to the unit vector of the sphere radius $\boldsymbol{p}_{2}$, i.e., $\boldsymbol{p}_{1} \cdot \boldsymbol{p}_{2}=0$ [26]. In order to ensure convergence of the solution of the nonlinear mechanical model, the discrete point $P_{d}$ is regarded as an element at the curved surface $G$. For the discrete point $P_{d}$, Eq. (55) can be employed; otherwise, Eq. (56) can be employed for $P_{i+1} \in U$. When $P_{i+1} \in U$, the curved surface may exert a contact distribution force $\boldsymbol{f}_{s p, i+1}$ on the cable along the same direction $\boldsymbol{p}_{2}$ as that of the sphere radius at the point $P_{i+1} \cdot f_{s p, i+1} \neq 0$ when the discrete point $P_{i+1}$ is on the curved surface $G$, whereas $f_{s p, i+1}=0$ when it is not on the curved surface $G$ [25]. By combining Eqs. (55) and (56), we obtain $n_{\mathrm{c}}$ equations, where $f_{s p, i+1}\left(t_{1} \leq i+1 \leq t_{1}+n_{\mathrm{c}}-1\right)$ are $n_{\mathrm{c}}$ Eq. (39) requires the addition of $n_{c}$ unknown varibles and $n_{c}$ equations (But in this paper, we have added $n$ unknown variables and $n$ equations in order to easily modify the calculation code under different calculation conditions. When $P_{i+1} \notin U$, the additional constraint added at the discrete point $P_{i+1}$ is: $f_{s p, i+1}-0=0$.). If $P_{i+1} \in U$, the force distribution in Eq. (33) needs to be modified according to Eq. (57).

In addition, the distribution forces at the calculation unit Part $i$ are concentrated at the discrete point $P_{i+1}$. As is shown in Fig. 12, $v_{t_{0}}$ is the direction of movement of the end point $P_{n+1}$ of the cable ( $\boldsymbol{v}_{t_{0}}$ is also perpendicular to the $Z$-axis in the coordinate system $O-X Y Z$ ), and the cable segment at discrete point $P_{1}$ is fixed. When the rotation angle of the orientation joint is relatively small, the movement of the cable at the $P_{1} H$ segment is also relatively small, so the movement at the discrete point $P_{i+1}$ can be simplified as the rotation of the point $P_{i+1}$ around the point $H$. Therefore, the relative motion trend $v_{t}$ at the discrete point $P_{i+1}$ is per-

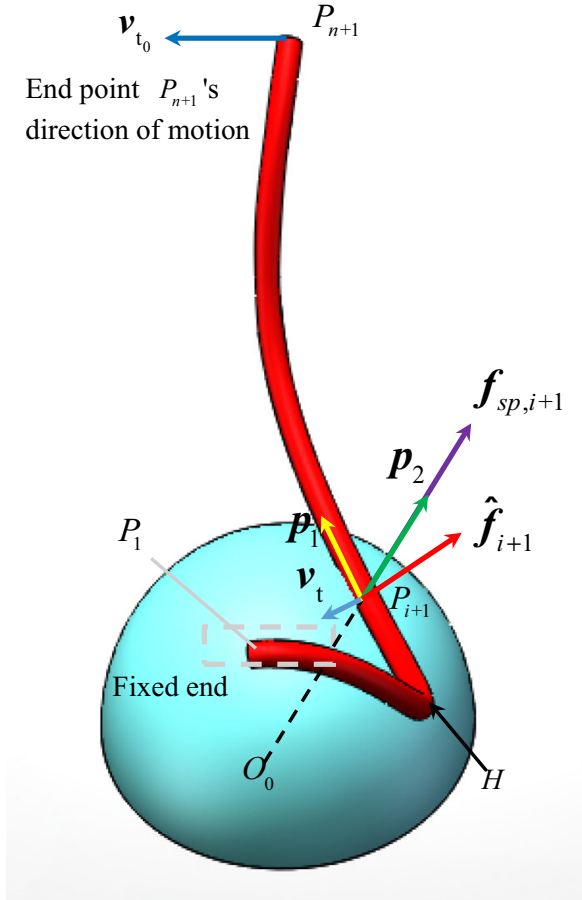

Fig. 12 Frictional constraint of the cable on a curved surface

pendicular to $\boldsymbol{p}_{1}$ and $\boldsymbol{p}_{2}$, which means that the direction of $\boldsymbol{v}_{t}$ can be determined; $\hat{\boldsymbol{f}}_{i+1}$ is the friction force distribution at the discrete point $P_{i+1}$ (Part $i$ ) that is oriented in the direction opposite that of $\boldsymbol{v}_{t}$. By neglecting the cable axial friction, we obtain $\mu_{\mathrm{c}}=0.35$, which is the friction coefficient between the cable and surface.

$\boldsymbol{p}_{1}=\frac{1}{\tilde{l}_{0}}\left[r_{X, i+1}-r_{X, i} r_{Y, i+1}-r_{Y, i} r_{Z, i+1}-r_{Z, i}\right]^{T}$

$\boldsymbol{p}_{2}=\frac{1}{R_{0}}\left[r_{X, i+1}-X_{0} r_{Y, i+1}-Y_{0} r_{Z, i+1}-Z_{0}\right]^{T}$

$\boldsymbol{f}_{s p, i+1}=f_{s p, i+1} \cdot \boldsymbol{p}_{2}$

$\hat{\boldsymbol{f}}_{i+1}=\mu_{\mathrm{c}}\left(\boldsymbol{p}_{1} \times \boldsymbol{f}_{s p, i+1}\right)$

The projection of $\hat{\boldsymbol{f}}_{i+1}$ onto the world coordinate system $O-X Y Z$ is given as 


$$
\begin{aligned}
& \hat{f}_{i+1}=\left[\begin{array}{lll}
\hat{f}_{X, i+1} & \hat{f}_{Y, i+1} & \hat{f}_{Z, i+1}
\end{array}\right]^{T} \\
& =\frac{\mu_{\mathrm{c}} \cdot f_{s p, i+1}}{\tilde{l}_{0} R_{0}}\left[\begin{array}{c}
\left(r_{Y, i+1}-r_{Y, i}\right)\left(r_{Z, i+1}-Z_{0}\right)-\left(r_{Z, i+1}-r_{Z, i}\right)\left(r_{Y, i+1}-Y_{0}\right) \\
\left(r_{Z, i+1}-r_{Z, i}\right)\left(r_{X, i+1}-X_{0}\right)-\left(r_{X, i+1}-r_{X, i}\right)\left(r_{Z, i+1}-Z_{0}\right) \\
\left(r_{X, i+1}-r_{X, i}\right)\left(r_{Y, i+1}-Y_{0}\right)-\left(r_{Y, i+1}-r_{Y, i}\right)\left(r_{X, i+1}-X_{0}\right)
\end{array}\right] \\
& g_{d}=\left|\sqrt{\left(r_{X, d}-X_{0}\right)^{2}+\left(r_{Y, d}-Y_{0}\right)^{2}+\left(r_{Z, d}-Z_{0}\right)^{2}}-R_{0}\right| \\
& +\left|\frac{\left(r_{X, d}-X_{0}\right)\left(r_{X, d}-r_{X, d-1}\right)+\left(r_{Y, d}-Y_{0}\right)\left(r_{Y, d}-r_{Y, d-1}\right)+\left(r_{Z, d}-Z_{0}\right)\left(r_{Z, d}-r_{Z, d-1}\right)}{\tilde{l}_{0} R_{0}}\right|=0 \\
& g_{i+1}=f_{s p, i+1}\left|\sqrt{\left(r_{X, i+1}-X_{0}\right)^{2}+\left(r_{Y, i+1}-Y_{0}\right)^{2}+\left(r_{Z, i+1}-Z_{0}\right)^{2}}-R_{0}\right| \\
& +f_{s p, i+1}\left|\frac{\left(r_{X, i+1}-X_{0}\right)\left(r_{X, i+1}-r_{X, i}\right)+\left(r_{Y, i+1}-Y_{0}\right)\left(r_{Y, i+1}-r_{Y, i}\right)+\left(r_{Z, i+1}-Z_{0}\right)\left(r_{Z, i+1}-r_{Z, i}\right)}{\tilde{l}_{0} R_{0}}\right|=0, \\
& \text { where }\left(i+1 \in\left[t_{1}, d\right) U\left(d, t_{1}+n_{c}-1\right]\right) \\
& {\left[\begin{array}{c}
f_{x, i+1} \\
f_{y, i+1} \\
f_{z, i+1}
\end{array}\right]={ }_{O}^{P_{i+1}} \boldsymbol{R}^{-1}\left[\begin{array}{c}
\hat{f}_{X, i+1}+f_{s p, i+1}\left(r_{X, i+1}-X_{0}\right) / R_{0} \\
\hat{f}_{Y, i+1}+f_{s p, i+1}\left(r_{Y, i+1}-Y_{0}\right) / R_{0} \\
\hat{f}_{Z, i+1}+f_{0}+f_{s p, i+1}\left(r_{Z, i+1}-Z_{0}\right) / R_{0}
\end{array}\right]}
\end{aligned}
$$

It is worth noting that $v$ provides $(7 n+11+$ $n_{\mathrm{c}}$ )-dimensional nonlinear overdetermined equations, which can be employed to obtain $7(n+1)+n_{\mathrm{c}}$ parameters (But in the calculation code of this paper, $\boldsymbol{v}$ is $(7 n+11+n)$-dimensional and we get $7(n+1)+n$ parameters.). According to the algorithm illustrated in Fig. 6, the nonlinear mechanical model of a cable with curved surface contact can be solved. When a spline curve is used to fit the discrete points, the simulated cable may slightly overlap the curved surface. Thus, the interpolation points at the curved surface have to be modified by using Eq. (58); $Z^{\prime}$ is the corrected coordinate value.

$$
\begin{aligned}
Z^{\prime}= & \frac{Z-Z_{0}}{\left|Z-Z_{0}\right|} \sqrt{R_{0}^{2}-\left(X-X_{0}\right)^{2}-\left(Y-Y_{0}\right)^{2}} \\
& +Z_{0}
\end{aligned}
$$

With the change in the rotation angle $\gamma$ of the orientation joint, the shape of the cable in contact with the curved surface can be obtained. Figure 13 shows the projections of the calculated trajectory of the cable, which is in contact with a curved surface, onto each coordinate plane. Figure 14 shows the comparison between the theoretical shape of the robot cable and the actual trajectory in contact with a curved surface. When $\gamma=\pi / 4, \pi / 3$ and $5 \pi / 12$, the robot cable comes into contact with the curved surface (spherical obstacle). The trends of the cable theoretical shape and the actual trajectory are consistent. Figure 15 shows the projection of the internal force on the end of the cable, which is in contact with the curved surface, in the coordinate system $P-x y z$. When $\gamma=\pi / 3$, due to the contact force of the curved surface, the end-point force (in Fig. 15) under the constraint of a curved surface is smaller than the end-point force under the restraint of both ends (in Fig. 10). This is consistent with the actual situation, and to a certain extent, it also verifies the correctness of the theoretical results. 

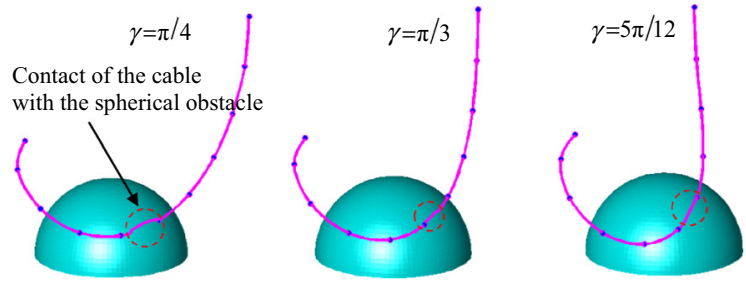

(a)

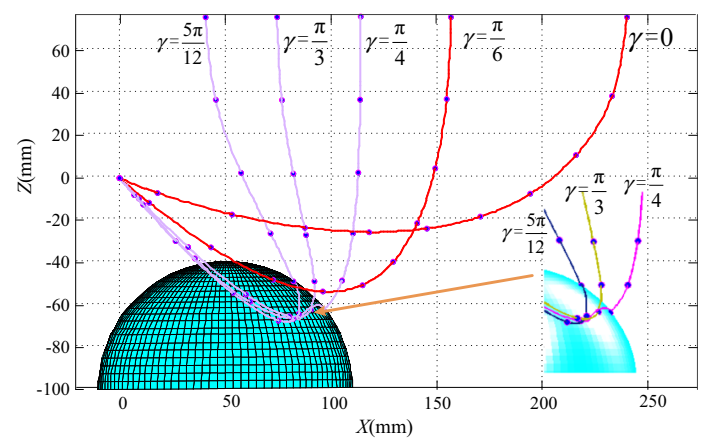

(b)

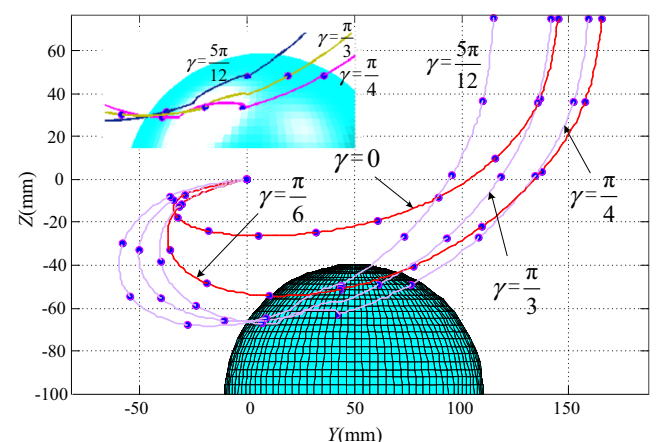

(c)

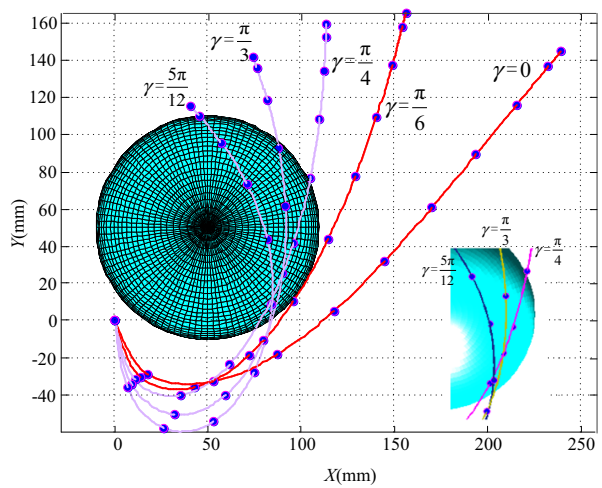

(d)

Fig. 13 Simulated shape of the cable in contact with a curved surface: a Contact of the cable with the spherical obstacle, projections onto the $\mathbf{b} X O Z$, $\mathbf{c} Y O Z$, and $\mathbf{d} X O Y$ planes

\section{Conclusion}

Under the complex conditions of multi-physics outer space environment, cables are the main sources of unexpected nonlinear disturbance effects on space robot joints. This induces slow rotation or oscillation of the joint at a certain position. In this study, by considering the gravitational force distribution, friction force distribution, and contact with the curved surface of a robot cable, we employ the mechanical parameters for each discrete point of the cable as variables and develop a discrete nonlinear mechanical model of a robot cable.

The following conclusions can be drawn:

1. In this paper, the nonlinear mechanical model of a cable in the space robotic arm is transformed into a large-scale nonlinear optimization problem, and the mechanical characteristics of each discrete point of the cable are systematically revealed including the end-point force and geometry of the cable. This model could be employed to facilitate stable operation of robotic arms designed for future outer space missions;

2. A solution algorithm, which ensures global convergence of the algorithm and is based on a trust-region adjustment strategy, is proposed to solve the nonlinear mechanical model of a cable. The rationality and accuracy of the proposed nonlinear mechanical cable model are verified by performing a morphological comparison;

3. At present, we use ordinary cables for our experiments. The next step is to use real space cables for experimental research. At the same time, we will further improve the experimental environment and make it as consistent as possible with the outer space environment. In addition, it is also important for future studies to theoretically determine the specific positions at which a robotic joint may exhibit slow rotation or oscillation, and to perform parallel light pipe experiments to estimate these positions on a micro-scale. 
Fig. 14 Comparison of theoretical shape and actual trajectory of the cable of a robot in contact with a curved surface

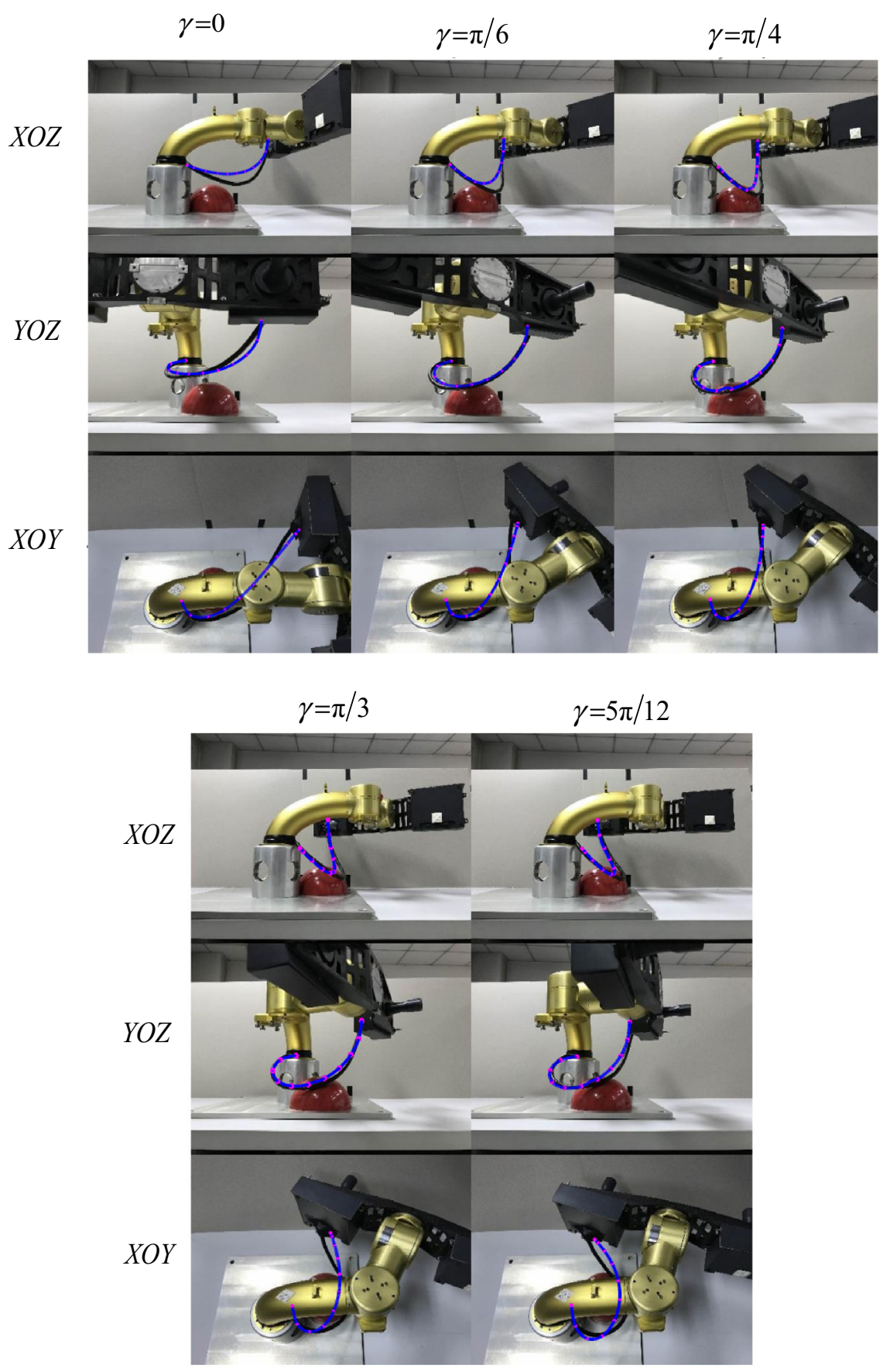




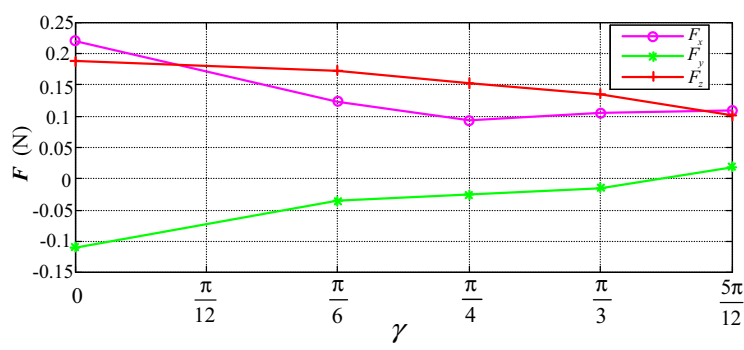

Fig. 15 Force at the end-point of the cable of a robot in contact with a curved surface

Acknowledgements The authors express their gratitude to the referees for very carefully reading and constructive comments and detailed suggestions which have improved this paper. This study is supported by the National Natural Science Foundation of China (51605474), State Key Laboratory of Robotics Fund (2016-Z09), and Innovation Foundation for National Defense Science and Technology of Chinese Academy of Sciences (CXJJ17-M109).

\section{Compliance with ethical standards}

Conflict of interest The authors declare that they have no conflict of interest.

Open Access This article is distributed under the terms of the Creative Commons Attribution 4.0 International License (http:// creativecommons.org/licenses/by/4.0/), which permits unrestricted use, distribution, and reproduction in any medium, provided you give appropriate credit to the original author(s) and the source, provide a link to the Creative Commons license, and indicate if changes were made.

\section{References}

1. Payandeh, S., Shi, F.: Interactive multi-modal suturing. Virtual Real. 14(4), 241-253 (2010)

2. Servin, M., Lacoursière, C.: Rigid body cable for virtual environments. IEEE Trans. Vis. Comput. Graph. 14(4), 783796 (2008)

3. Lazarus, A., Miller, J.T., Reis, P.M.: Continuation of equilibria and stability of slender elastic rods using an asymptotic numerical method. J. Mech. Phys. Solids 61(8), 1712-1736 (2013)

4. Kumar, A., Kumar, S., Gupta, P.: A helical Cauchy-Born rule for special Cosserat rod modeling of nano and continuum rods. J. Elast. 124(1), 81-106 (2016)

5. Gao, A., Murphy, R.J., Liu, H., Iordachita, I.I., Armand, M.: Mechanical model of dexterous continuum manipulators with compliant joints and tendon/external force interactions. IEEE ASME Trans. Mechatron. 22(1), 465-475 (2017)

6. Heijden, G.H.M.V.D., Thompson, J.M.T.: Helical and localised buckling in twisted rods: a unified analysis of the symmetric case. Nonlinear Dyn. 21(1), 71-99 (2000)

7. Goriely, A., Tabor, M.: The nonlinear dynamics of filaments. Nonlinear Dyn. 21(1), 101-133 (2000)
8. Lafortune, S., Goriely, A., Tabor, M.: The dynamics of stretchable rods in the inertial case. Nonlinear Dyn. 43(1-2), 173-195 (2006)

9. Love, A.E.H.: A Treatise on the Mathematical Theory of Elasticity, 4th edn. Dover Publications, New York (1944)

10. Dill, E.H.: Kirchhoff's theory of rods. Arch. Hist. Exact Sci. 44(1), 1-23 (1992)

11. Wang, P., Xue, Y.: Conformal invariance of Mei symmetry and conserved quantities of Lagrange equation of thin elastic rod. Nonlinear Dyn. 83(4), 1815-1822 (2016)

12. Weiss, H.: Dynamics of geometrically nonlinear rods: I. Mechanical models and equations of motion. Nonlinear Dyn. 30(4), 357-381 (2002)

13. Xue, Y., Liu, Y., Chen, L.: Methods of analytical mechanics for dynamics of the Kirchhoff elastic rod. Acta Phys. Sin. 55(8), 3845-3851 (2006). (in Chinese)

14. Greco, L., Cuomo, M.: B-Spline interpolation of KirchhoffLove space rods. Comput. Meth. Appl. Mech. Eng. 256, 251-269 (2013)

15. Ram, Y.M.: Nonlinear eigenvalue problems of the elastic. Mech. Syst. Signal Process. 45(2), 408-423 (2014)

16. Kratchman, L.B., Bruns, T.L., Abbott, J.J., Webster, R.J.: Guiding elastic rods with a robot-manipulated magnet for medical applications. IEEE Trans. Robot. 33(1), 227-233 (2017)

17. Luo, M., Xie, H., Xie, L., Cai, P., Gu, L.: A robust and realtime vascular intervention simulation based on Kirchhoff elastic rod. Comput. Med. Imaging Graph. 38(8), 735-743 (2014)

18. Bretl, T., Mccarthy, Z.: Quasi-static manipulation of a Kirchhoff elastic rod based on a geometric analysis of equilibrium configurations. Int. J. Robot. Res. 33(1), 48-68 (2014)

19. Wang, Z., Fratarcangeli, M., Ruimi, A., Srinivasa, A.R.: Real time simulation of inextensible surgical thread using a Kirchhoff rod model with force output for haptic feedback applications. Int. J. Solids Struct. 113-114, 192-208 (2017)

20. Lillian, T.D., Taranova, M., Wereszczynski, J., Andricioaei, I., Perkins, N.C.: A multiscale dynamic model of DNA supercoil relaxation by topoisomerase IB. Biophys. J. 100(8), 2016-2023 (2011)

21. Olson, S.D., Lim, S., Cortez, R.: Modeling the dynamics of an elastic rod with intrinsic curvature and twist using a regularized Stokes formulation. J. Comput. Phys. 238, 169187 (2013)

22. Liu, Y.: Nonlinear Mechanics of Thin Elastic Rod: Theoretical Basis of Mechanical Model of DNA, 1st edn. Tsinghua University Press, Beijing (2006). (in Chinese)

23. Liu, J., Zhao, T., Wang, C., Tang, C.: Motional cable harness physical characteristic oriented modeling and kinetic simulation technology in virtual environment. J. Mech. Eng. 47(9), 117-124 (2011). (in Chinese)

24. Liu, J., Zhao, T., Ning, R., Liu, J.: Physics-based modeling and simulation for motional cable harness design. Chin. J. Mech. Eng. 27(5), 1075-1082 (2014)

25. Jin, W., Liu, J., Liu, J., Tang, C., Lin, H.: Motional cable harness physical characteristic oriented modeling and kinetic simulation technology under smooth plane constraints. J. Mech. Eng. 52(3), 118-127 (2016). (in Chinese)

26. Xue, Y., Chen, L., Liu, Y.: Problems on equilibrium of a thin elastic rod constrained on a surface. Acta Phys. Sin. 53(7), 2040-2045 (2004). (in Chinese) 
27. Wang, C., Ning, R., Liu, J., Zhao, T.: Dynamic simulation and disturbance torque analyzing of motional cable harness based on Kirchhoff rod model. Chin. J. Mech. Eng. 25(2), 346-354 (2012)

28. Korayem, M.H., Tourajizadeh, H.: Maximum DLCC of spatial cable robot for a predefined trajectory within the workspace using closed loop optimal control approach. J. Intell. Robot. Syst. 63(1), 75-99 (2011)

29. He, W., Ge, S.S.: Cooperative control of a nonuniform gantry crane with constrained tension. Automatica 66, 146-154 (2016)

30. Korayem, M.H., Tourajizadeh, H., Zehfroosh, A., Korayem, A.H.: Optimal path planning of a cable-suspended robot with moving boundary using optimal feedback linearization approach. Nonlinear Dyn. 78(2), 1515-1543 (2014)

31. Korayem, M.H., Tourajizadeh, H., Bamdad, M.: Dynamic load carrying capacity of flexible cable suspended robot: robust feedback linearization control approach. J. Intell. Robot. Syst. 60(3-4), 341-363 (2010)
32. Balaeff, A., Mahadevan, L., Schulten, K.: Modeling DNA loops using the theory of elasticity. Phys. Rev. E 73(1), 031919 (2003)

33. Whittaker, E.T.: A Treatise on the Analytical Dynamics of Particles and Rigid Bodies, with an Introduction to the Problem of Three Bodies, 4th edn. Cambridge University Press, Cambridge (1960)

34. He, W., Ouyang, Y., Hong, J.: Vibration control of a flexible robotic manipulator in the presence of input deadzone. IEEE Trans. Ind. Inform. 13(1), 48-59 (2016)

35. He, W., He, X., Zou, M., Li, H.: PDE model-based boundary control design for a flexible robotic manipulator with input backlash. IEEE Trans. Control Syst. Technol. (2018). https:// doi.org/10.1109/TCST.2017.2780055 